\title{
Clinical features of a novel TIMP-3 mutation causing Sorsby's fundus dystrophy: implications for disease mechanism
}

Michael Clarke, Keith W Mitchell, Judith Goodship, Sharon McDonnell, Michael D Barker, Ian D Griffiths, Norman McKie

\begin{abstract}
Aims-To describe the phenotype in three family members affected by a novel mutation in the gene coding for the enzyme tissue inhibitor of metalloproteinase-3 (TIMP-3).

Methods-Three members of the same family were seen with a history of nyctalopia and visual loss due to maculopathy. Clinical features were consistent with Sorsby's fundus dystrophy. Exon 5 of the gene coding for TIMP-3 was amplified by the polymerase chain reaction, single strand conformation polymorphism analysis undertaken and exon 5 amplicons were directly sequenced.

Results-Onset of symptoms was in the third to fourth decade. Five of six eyes had geographic macular atrophy rather than neovascularisation as a cause for central visual loss. Peripheral retinal pigmentary disturbances were present. Scotopic ERGs were abnormal in all three. Mutation analysis showed a $\mathbf{G} \rightarrow T$ transversion in all three resulting in a premature termination codon, E139X, deleting most of the carboxy terminal domain of TIMP-3. Conclusions-The patients described had a form of Sorsby's fundus dystrophy which fell at the severe end of the spectrum of this disease. Postulated disease mechanisms include deposition of dimerised TIMP-3 protein.

(Br F Ophthalmol 2001;85:1429-1431)
\end{abstract}

Service, Newcastle upon Tyne, UK

J Goodship

S McDonnell

Division of Oncology and Cellular Pathology, Pathology Section, University of Sheffield, Medical School, Sheffield SR10

2RX, UK

M D Barker

Department of

Rheumatology,

University of

Newcastle upon Tyne,

Newcastle upon Tyne

NE2 4HH, UK

I D Griffiths

$\mathrm{N}$ McKie

Correspondence to: M Clarke

M.P.Clarke@ncl.ac.uk

Accepted for publication 11 July 2001
Japanese family has been described with a splice site mutation. ${ }^{7}$ All UK families had been thought to have a common S181C mutation, indicating a founder effect ${ }^{8}$; however, we have recently identified a family from the north east of England with a previously undescribed mutation causing a premature termination codon and loss of the terminal 49 amino acids of the protein.

\section{Case descriptions}

Patient III: 2 presented with loss of central acuity and night blindness at the age of 25 . When examined at the age of 72 , the visual acuity in each eye was hand movements only. There was complete chorioretinal atrophy at each macula. The retinal vessels were thinned and there was pigmentation in the retinal periphery.

Rheumatological and general medical examination was normal apart from varicose veins and mild discomfort in the hip and shoulder. Renal function was normal.

Patient IV:4 presented aged 36 years with blurred vision in the left eye. Visual acuity was $6 / 6$ in the right eye and 6/60 in the left eye. Examination of the left retina showed a disciform scar. Drusen were present at the right macula and there was peripheral retinal pigmentation in both eyes. An electroretinogram showed photopic responses which were within normal limits (although those from the right eye were larger than from the left), but scotopic responses were subnormal in both eyes.An electro-oculogram had an Arden ratio of 1.2 indicating retinal pigment epithelial dysfunction. At age 44 years the vision in the right eye deteriorated over a period of several weeks to $6 / 24$. Examination showed a flat pigmentary disturbance which did not leak on fluorescein angiography (Fig 1).

Rheumatological and general medical examination was normal. Creatinine was $116 \mu \mathrm{mol} / 1$ (normal less than 110), urea $5.2 \mathrm{mmol} / \mathrm{l}$.

Patient IV:10 presented aged 37 with a 4 year history of nyctalopia predominantly affecting central vision. Her own mother, the sister of case 1 had died in early middle age without developing visual symptoms.

Visual acuity at presentation was $6 / 18$ right and 6/9 left with a low myopic correction. Retinal examination was within normal limits apart from peripheral choroidal hypopigmentation. An electroretinogram showed normal photopic responses but on dark adaptation the scotopic b waves were broad and of low amplitude and did not increase on prolonged adaptation, indicating a defect in the rod photoreceptor system 

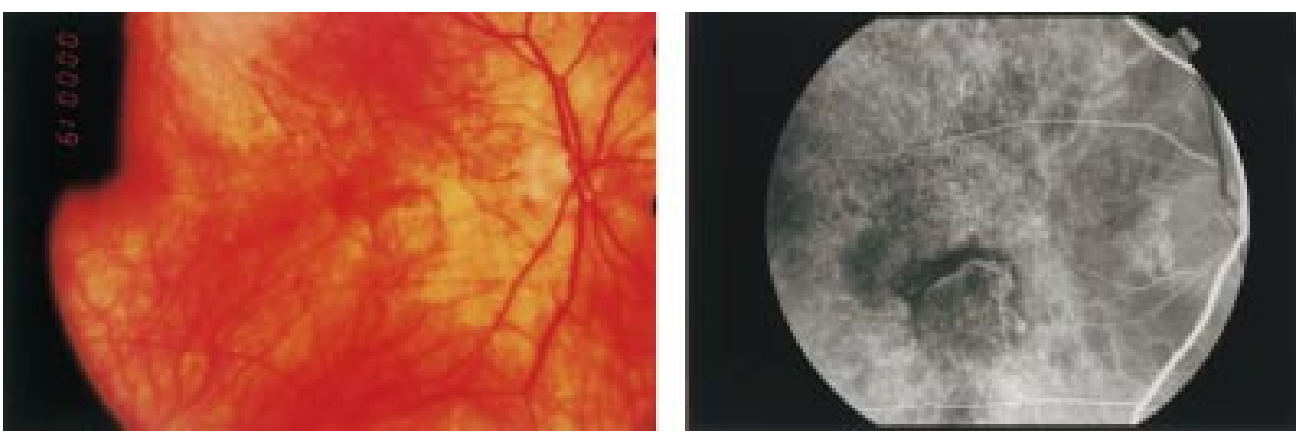

Figure 1 Patient IV:4. (A) Right fundus. (B) Right fundus fluorescein angiogram, transit phase.

(Fig 2). Pattern electroretinograms were totally extinguished in right eye, and just recordable in the left.

A fluorescein angiogram showed paramacular defects of the retinal pigment epithelium. Subsequently, geographic atrophy of the retinal pigment epithelium and choroid at the macula led to loss of central vision 17 years after presentation.

Rheumatological and general medical examination was normal apart from mild varicose veins.

\section{DNA analysis}

Blood was obtained from the three affected individuals and a normal control, and polymerase chain reaction, restriction digest, single strand conformation polymorphism analysis, and DNA sequencing were carried out for exon 5 of the TIMP-3 gene as described previously. ${ }^{9}$ Direct DNA sequencing showed a $\mathrm{G} \rightarrow \mathrm{T}$ transversion in one allele in the first base of codon 139 in the three affected individuals resulting in a TAG termination codon replacing the normal GAG glutamic acid codon (E139X). They did not have the S181C mutation seen in other British families.

\section{Discussion}

Sorsby's fundus dystrophy is known to be associated with mutations in the gene for TIMP- $3 .{ }^{4}$ There are four very similar tissue inhibitors of metalloproteinases (TIMPs) $^{10}$ comprising a gene family with 12 highly conserved cysteine residues which form six disulphide bridges

Male control, age 46, LE ERG Sorsby's patient, age 52, LE ERG
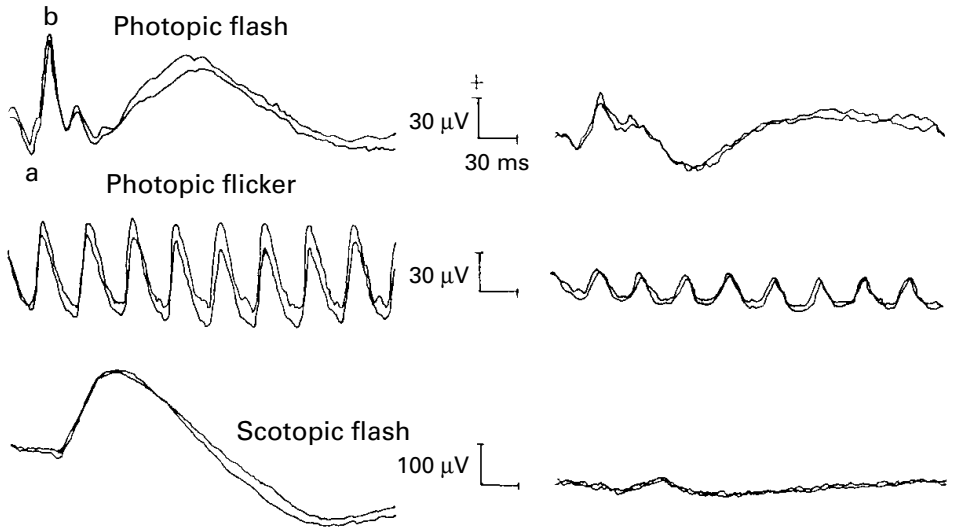

Figure 2 Photopic flash and flicker; and scotopic flash full field ERGs of patient IV:10 and age matched control. essential for correct protein folding and function. TIMPs consist of two domains each stabilised by three disulphide bonds, an amino terminal inhibitory domain, through which they bind to an active matrix metalloproteinase, and a carboxy terminal domain, which is involved in interactions with proform matrix metalloproteinases. The positions of the disulphide bonds have been assigned in TIMP-1 and by comparison of the two molecules it is likely that the disulphide bonds in the carboxy terminus of TIMP-3 are between residues 122-169, 127132, and 140-161. ${ }^{11}$ Immunohistochemistry of human eyes shows TIMP-3 specific staining in Bruch's membrane, particularly in the basement membranes of retinal pigment epithelial and endothelial cells. ${ }^{12}$ Overexpression of TIMP-3 in transfected rat retinal pigment epithelium inhibits experimental choroidal neovascularisation. ${ }^{13}$

The five mutations previously identified in patients with Sorsby's fundus dystrophy are in the carboxy terminal domain and all lead to cysteine substitutions (S156C, G166C, G167C, Y168C, S181C). It is likely that these cysteine substitutions lead to abnormal disulphide bonding and thus alter the protein's conformation. The novel mutation we have identified deletes most of the carboxy terminal domain and will be expected to alter its structure markedly as only one of the disulphide bridges could form. This mutation leaves the C-terminal region in TIMP-3 with an unpaired cysteine residue by deleting three of the six cysteines and so may also lead to altered protein conformation.

Ocular features in pedigrees with SFD have shown considerable interfamilial, intrafamilial, and even intraindividual variations with regard to age of onset, speed of progression, involvement of the second eye, neovascularisation, and peripheral retinal involvement. ${ }^{14}$

Only one member of our family had a disciform scar in one eye. In families with the S181C, Y168C, and S156C mutations, neovascularisation is a prominent feature. ${ }^{5}{ }^{15}$ Peripheral visual field loss is seen in both the S181C and E139X mutations, although night blindness tended to precede visual loss in individuals with the E139X mutation. A Japanese family has been described with a splice site mutation which causes a later onset (seventh decade) disease with preserved peripheral retinal function. ${ }^{7}$

The mechanism by which mutations in TIMP-3 give rise to retinal disease is not 
known, but is unlikely to be due to loss of TIMP-3 function as expression studies have shown all known disease causing mutants to be functional metalloproteinase inhibitors. ${ }^{9}$ An eye from a patient with SFD has been studied histologically, and has been shown to have an eosinophilic deposit at the level of Bruch's membrane. ${ }^{2}$ This deposit stains for TIMP- $3^{16}$ and may represent an insoluble dimerised form of the enzyme produced by abnormal disulphide bond formation by the mutated protein which may be associated with elastin. ${ }^{17} \mathrm{We}$ have previously demonstrated dimerisation of the protein product of S181C, S156C, G166C, and E139X. ${ }^{9}$ One possible mechanism for retinal disease may therefore be impairment of the nutrition and metabolism of the outer retina because of abnormal deposition of dimerised TIMP-3 in Bruch's membrane. This is supported by the finding of reversal of night blindness in SFD using high dose vitamin A supplementation. ${ }^{18}$ TIMP-3 is known to express apoptotic properties when overexpressed in vitro, ${ }^{19}$ and an altenative mechanism, particularly for the patients' atrophic maculopathy, could be apoptosis of photoreceptor and retinal pigment epithelial cells induced by accumulation of dimerised TIMP-3.

This work was supported in part by the special trustees of the Newcastle University Hospitals. Mr SJ Talks provided valuable advice about the retinal findings in these patients.

1 Sorsby A, Mason ME. A fundus dystrophy with unusual features. Br f Ophthalmol 1949;33:67-97.

2 Capon M, Marshall J, Krafft J, et al. Sorsby's fundus dystrophy: a light and electron microscopic study. Ophthal dystrophy: a light and electro

3 J Evans, C Rooney, F Ashwood, et al. Blindness and partial sight in England and wales: April 1990-March 1991. Health Trends 1996;28:5-12

4 Weber BHF, Vogt G, Pruett RC, et al. Mutations in the tissue inhibitor of metalloproteinases-3 (TIMP3) in patients with Sorsby's fundus dystrophy. Nat Genet 1994;8:352-6.
5 Felbor U, Stöhr H, Amann T, et al. A novel Ser156Cys mutation in the tissue inhibitor of metalloproteinases-3 mutation in the tissue inhibitor of metalloproteinases-3
(TIMP-3) in Sorsby's fundus dystrophy with unusual clini(TIMP-3) in Sorsby's fundus dystrophy with un

6 Felbor U, Suvanto EA, Forsius HR, et al. Autosomal recessive Sorsby fundus dystrophy revisited: molecular evidence for dominant inheritance. Am f Hum Genet. 1997;60:5762.

7 Tabata Y, Isashiki Y, Kamimura K, et al. A novel splice site mutation in the tissue inhibitor of metalloproteinases-3 mutation in the tissue inhibitor of metalloproteinases-3 features. Hum Genet 1998;103:179-82.

8 Wijesuriya SD, Evans, K, Jay MR, et al. Sorsby's fundus dystrophy in the British Isles: demonstration of a striking founder effect by microsatellite-generated haplotypes. Genome Res 1996; 6:92-101.

9 Langton KP, McKie N, Curtis A, et al. A novel tissue inhibitor of metalloproteinases-3 mutation reveals a common molecular phenotype in Sorsby's fundus dystrophy. $\mathcal{F}$ Biol Chem 2000;275:27027-31.

10 Apte SS, Olsen BR, Murphy G. The gene structure of tissue inhibitor of metalloproteinases (TIMP) -3 and its inhibitory activities define the distinct TIMP gene family. $\mathcal{F}$ Biol Chem 1995;270:14313-18.

11 Williamson RA, Marston FAO, Angal S, et al. Disulphide bond assignment in human tissue inhibitor of metalloproteinases (TIMP). Biochem f 1990;268:267-74.

12 Vranka JA, Johnson E, Zhu X, et al. Discrete expression and distribution pattern of TIMP-3 in the human retina and choroid. Curr Eye Res 1997;16:102-10.

13 Takahashi T, Nakamura T, Hayashi A, et al. Inhibition of experimental choroidal neovascularisation by overexpression of tissue inhibitor of metalloproteinases-3 in retinal pigment epithelial cells. Am f Ophthalmol 2000;130:77481

14 Felbor U, Benkwitz C, Klein ML, et al. Sorsby fundus dystrophy: reevaluation of variable expressivity in patients carrying a TIMP3 founder mutation. Arch Ophthalmol 1997;115:1569-71.

15 Hoskin A, Sehmi K, Bird AC. Sorsby's pseudoinflammatory macular dystrophy. Br 7 Ophthalmol 1981;65:859-65.

16 Fariss RN, Apte SS, Luthert PJ, et al. Accumulation of tissue inhibitor of metalloproteinases- 3 in human eyes with Sorsby's fundus dystrophy or retinitis pigmentosa. $\mathrm{Br} f$ Ophthalmol 1998;82:1329-34

17 Chong NHV, Alexander RA, Gin T, et al. TIMP-3, collagen, and elastin immunohistochemistry and histopathology of Sorsby's fundus dystrophy. Invest Ophthalmol Vis Sci 2000; 41:898-902.

18 Jacobson SG, Cideciyan AV, Regunath G, et al. Night blindness in Sorsby's fundus dystrophy reversed by vitamin A. Nat Genet 1995;11:27-32.

19 Baker AH, Zaltsman AB, George SJ, et al. Divergent effects of tissue inhibitor of metalloproteinase- $1,-2$, or -3 overexpression on rat vascular smooth muscle cell invasion, proliferation, and death in vitro. F Clin Invest 1998;101: $1478-87$ 